\title{
EL NIVEL DE IMPLICACIÓN DEL CONSUMIDOR CON EL DEPORTE Y SU \\ INFLUENCIA SOBRE LA IMAGEN DEL ESPONSOR Y EL EVENTO, Y LA INTENCIÓN DE COMPRA EN EL PATROCINIO DEPORTIVO.
}

\author{
Zapata Agüera, Soledad ${ }^{1}$ \\ Martínez Caro, Laura ${ }^{2}$ \\ Universidad Politécnica de Cartagena \\ soledad.zapata@upct.es
}

laura.martinez@upct.es

Material original autorizado para su primera publicación en la revista académica REDMARKA. Revista Digital de Marketing Aplicado. https://doi.org/10.17979/redma.2017.02.019.4855

Recibido: 28 Junio 2017

Aceptado: 29 Octubre 2017

\footnotetext{
${ }^{1}$ Licenciada en Publicidad y Relaciones Públicas. Profesora de la Universidad Politécnica de Cartagena, desarrollando esta actividad desde el año 2001. Actividad investigadora enmarcada dentro del campo del patrocinio deportivo y el comportamiento del consumidor, publicando en el Anuario de Jóvenes Investigadores, así como realizando diferentes ponencias en Congreso Internacional de Educación Física, Congreso Internacional de Fútbol Base, Encuentro de Profesores Universitarios de Marketing, Congreso EBES (Eurasia Business and Economics Society,. Actualmente desarrollando la tesis sobre esponsorización deportiva.Departamento de Economía de la Empresa, Calle Real 3 - 30201. Cartagena. Murcia. España, ${ }^{2}$ Profesora del Área de Comercialización e Investigación de Mercados de la Universidad Politécnica de Cartagena. Su actividad investigadora se enmarca dentro del marketing de servicios y calidad, posee dos sexenios de investigación, y ha publicado numerosos estudios al respecto en revistas indexadas en rankings prestigiosos (Tourism Management, Journal Business Research, International Journal of Market Research, etc.). Ha participado en numerosos congresos y ha formado parte de varios proyectos de investigación. Además ha sido invitada a revisar artículos en revistas prestigiosas como The Service Industries Journal o International Journal of Management Science and Engineering Management.Departamento de Economía de la Empresa, Calle Real 3 - 30201. Cartagena. Murcia. España,
}

REDMARKA IMARKA-Universidad de A Coruña - CIECID Año X, Número 19, (2017), vol. 2 pp. 83-108 http://www.redmarka.net/ ISSN 1852-2300 


\section{Abstract}

In this investigation, we have the intention of demonstrating the influence of the consumer's involvement with the sport about a larger probability of seeing events related to this sport, the protector's most positive image and of the event, and more probability of buying the products or the sponsor's services. Because we used this self-administered questionnaire. The analyses were carried out with spas 15.0, checking the trust and dimensionality of the scales used. The contrast of the hypotheses was taken to-students out and in all of the hypotheses we obtained the acceptance of these. We can sustain, then that a high sport involvement of the consumer influences about the possibility of to attend or to see event related to that sport, very necessary for the exhibition for the sport sponsorship; it also influences the image of the event that is decisive in the formation of the sponsor's image, and finally, in the purchase intention that is a measure of the success of the sport sponsorship.

Keywords: Involvement, sponsorship and image

\section{Resumo}

Nesta investigação, nós temos a intenção de demonstrar a influência da implicação do consumidor com o esporte sobre uma probabilidade maior de ver eventos relacionou com este esporte, a imagem mais positiva do protetor e do evento, e mais probabilidade de comprar os produtos ou os serviços do protetor. Porque nós usamos isto autoadministrados de questionários. As análises foram levadas a cabo com Spss 15.0, conferindo a confiança e dimensionalidade das balanças usadas. $\mathrm{O}$ contraste das hipóteses esboçadas foi levado fora t-estudantes e em todas as hipóteses nós obtivemos a aceitação destes. Nós podemos sustentar, então que uma implicação maior nível com o esporte por parte do consumidor influencia sobre a possibilidade de assistir ou ver evento relacionou com aquele esporte, muito necessário para a exibição para o patronato desportivo; também influencia na imagem do evento que é decisivo na formação da imagem do protetor, e ultimamente, na intenção de compra que é uma medida muito efetiva do sucesso do patronato desportivo.

Palavras-chave: implicação, patronato, imagem 


\section{Introducción}

En las sociedades actuales, el aumento del tiempo de ocio, del grado cultural y del poder adquisitivo han desempeñado un papel fundamental para convertir el deporte en un estilo de vida y un modelo a seguir. Por ello, el consumo masivo de espectáculos y eventos deportivos ha ido cobrando, progresivamente, una gran relevancia, manifestando su protagonismo, especialmente en los medios de comunicación (García y Llopis, 2006).

Este aumento del consumo espectáculos y eventos deportivos ha provocado en los individuos una mayor implicación con este tipo de entretenimientos La estudios que analizan la variable implicación, han llegado a considerarla como una de las más importantes (Martin, 1998) y de mayor valor predictivo respecto al comportamiento de compra del consumidor (Evrard y Aurier, 1996). Además, destacan su influencia en el procesamiento de los datos almacenados en la memoria (Maheswaran y Sternthal, 1990) y en la búsqueda externa de información pre-compra (Chaudhuri, 2000).

En las últimas décadas, la conducta del consumidor deportivo ha sido objetivo de numerosos estudios (Capella, 2002; Galván y González, 2003; Gwinner y Swanson, 2003; Greenwood et al., 2006; Kim et al., 2008), surgiendo diferentes teorías desde las disciplinas de la psicología, la sociología y el marketing, que buscan explicar los fenómenos asociados a la conducta de este tipo de consumidor, y desarrollar y adaptar escalas que permitan medir diferentes aspectos tales como el reconocimiento, la actitud, la imagen, etc., relacionados con dicha conducta (Fisher y Wakefield, 1998; ; Shank y Beasley, 1998; Laverie y Arnett, 2000; Wann y Schrader, 2000; Capella, 2002).

La investigación que aquí se presenta pretende mostrar como la implicación del consumidor con un deporte media en la predisposición de éste a ver o consumir más eventos relacionados con ese deporte, e influye en determinados aspectos del patrocinio deportivo como son obtener un mejor reconocimiento del patrocinador, tener una percepción más favorable de éste y del evento, y mostrar una mayor disposición a comprar los productos patrocinados. 


\section{Investigación en la esponsorización: revisión de la literatura y planteamiento} de hipótesis.

\subsection{Implicación del consumidor y su relación con otras variables que influyen en patrocinio deportivo}

Los primeros en emplear el término "implicación" fueron Sherif y Cantril (1947 relacionando esta variable con el auto-concepto y el ego, y su influencia sobre las actitudes para posteriormente extenderse al estudio del comportamiento del consumidor.

En términos generales, la implicación tiene que ver con la importancia que un individuo le otorga a un objeto en particular (Greenwald y Leavitt, 1984). En este sentido, según Zaichowsky (1985, p. 342), la implicación es definida como "la importancia personal percibida hacia un objeto en particular, basada en sus necesidades, valores e intereses". Este mismo planteamiento es el suscrito en el campo del marketing deportivo por autores como Bennett et al. (2009); Ko et al. (2010); Beaton et al. (2011), Hur et al. (2012) o Stevens y Rosenberger (2012).

Lardinoit y Derbaix (2001, p.170) la definieron como "una especie de entusiasmo genuino, un interés fuerte y sólido, que viene a dar importancia a un objeto, actividad o persona para el individuo". Por su parte, Havitz y Dimanche (1997, p. 246) consideraban la implicación como "un estado inobservable de motivación, excitación o interés hacia una actividad recreativa o asociada a un producto".

Cuando esta excitación o interés se refiere a un estado motivacional, se pone de manifiesto en la relación al producto o con el acto de la compra, que van en función del sistema de valores del individuo (Laurent y Kapferer, 1985; Mittal, 1989; Day et al., 1995). Por lo tanto, la implicación incluye una valoración de la importancia del estímulo para el consumidor, pero añade un cierto comportamiento como consecuencia de la misma, es decir, la implicación motiva a la acción (Pérez, 2006).

De igual forma, cuando los consumidores participan en un mayor grado en un determinado evento, el procesamiento de la información se hace de manera central (Droge, 1989). Así en las comparaciones directas tiene una influencia positiva, ya que se ha producido por el tratamiento central de la información, y por lo tanto no hay ninguna interferencia debido a las motivaciones. Esta situación es más probable 
que surja cuando la implicación cognitiva es alta (una condición que aumenta el valor de la información comparativa para el consumidor) y hay una alta implicación afectiva. Además las personas procesan el mensaje de manera diferente dependiendo, entre otras variables, de su implicación personal con el mensaje, el producto o la decisión de compra. En definitiva, la implicación personal es el mayor determinante de la probabilidad de que el consumidor procese el mensaje (Funk, Ridinger y Moorman, 2004; Grohs y Reisinger, 2014).

Teniendo en cuenta lo anterior, Stone (1984) clasificó la implicación en dos estados: mental y comportamental (tal y como se muestra el diagrama de árbol representado en la Figura 1). El estado mental, tiene las siguientes ramificaciones: a) la "amplitud", entendida como la extensión de las asociaciones entre el conocimiento del estímulo y el propio conocimiento del individuo, y está representada por el número de valores asociados; b) la "relevancia", que indica la posición jerárquica de los conocimientos del individuo; y c) la "dirección e intensidad", que supone la fortaleza de la asociación, referida a la intensidad de tales conexiones. En cuanto al estado comportamental, podemos observar que se ramifica en tres aspectos: "búsqueda", "examen" y "argumentación". Estos últimos son la muestra de los tipos de comportamientos que indican grados de implicación conductual, por ejemplo más búsqueda de información indicaría mayor implicación por parte del consumidor.

Figura 1: Estados de la implicación

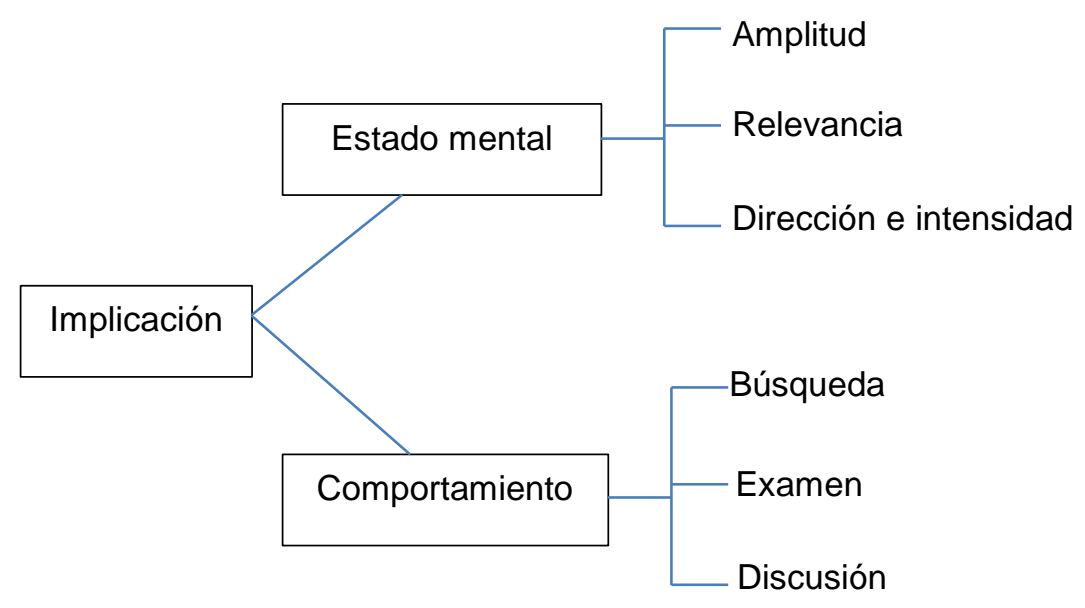

Fuente: Stone (1984) 
De forma similar a Stone (1984), pero concretamente en el ámbito deportivo, Bennett et al. (2009) distinguieron tres tipos de implicación: duradera, situacional, y comportamental (Richins et al, 1992; Day et al., 1995). En la implicación duradera, se da cuando un individuo compra la equipación y el vestuario asociado con el deporte de interés y además también consume el deporte a través de los medios de comunicación. Por lo tanto, un individuo que participe en un deporte será más probable que dedique más tiempo al consumo del mismo, asistiendo a eventos deportivos y viendo su retransmisión por televisión (Kahle et al., 1996; Laverie y Arnett, 2000; Donovan, Garlson, y Zimmerman, 2005). La implicación situacional, es aquella donde el individuo experimenta una implicación temporal (Rotchschild, 1984); en este caso la implicación está causada por la situación concreta en la que se encuentra el consumidor, además de estar influido tanto por el contexto físico como social de éste (Peter y Olson, 1987). Por último, la participación de comportamiento se refiere al tiempo y/o intensidad del esfuerzo gastado en la búsqueda de una actividad en particular (Stone, 1984).

Asimismo, a lo largo de la literatura, son varios los autores que han analizado los distintos niveles o grados de la implicación. Realizar esta diferenciación en distintos grados en el ámbito deportivo es importante, porque de acuerdo con Gwinner y Swanson (2003), el nivel de la implicación del consumidor con una actividad, persona o equipo esponsorizado, influye en la efectividad de la esponsorización. Así, el estudio realizado Meenaghan (2001) considera que los individuos no tienen un mismo nivel de implicación, y distingue tres niveles: 1) ligeramente implicado; 2) moderadamente implicado; y 3) altamente implicado. Meenaghan (2001) realiza esta clasificación para segmentar la población que visualiza un evento, y lo hace en base que esta no es homogénea en términos de grado de interés o participación.

En esta misma línea se muestran Dees et al. (2008), para quienes la implicación del fan es una construcción integral, lo que supone que al evaluar la eficacia de la esponsorización, debemos tener en cuenta que existen diferentes niveles emocionales de compromiso por parte del consumidor hacia el evento esponsorizado. Dependiendo de cuan implicado esté el consumidor con su deporte favorito o con el tipo de evento, éste podrá establecer diferentes relaciones con la esponsorización (Quester y Thompson, 2001). 
Este hecho es apoyado por Lascu et al. (1995), quienes sostienen que grandes niveles de implicación podrían afectar positivamente a la motivación del consumidor para procesar la información que recibe respecto de una actividad, equipo o deportista esponsorizado (Greenwald y Leavitt, 1984; Celsi y Olson, 1988). Por tanto, a mayor motivación en el procesado de información, mayor sería también la efectividad de la esponsorización.

Otros autores, consideran que esta mayor efectividad asociada a la implicación no solo implicaría un mayor reconocimiento del esponsor, como indicaba el estudio de Lascu et al. (1995), sino que abarcaría también a otras variables. Por ejemplo, Pham (1992), D'Astous y Bitz (1995) y Alexandris et al (2007), consideran que cuando la implicación del consumidor con un evento esponsorizado es alta, se genera una mayor simpatía hacia el esponsor y en consecuencia una imagen más positiva de éste. Asimismo, Levin et al. (2001) comprobaron que los individuos altamente implicados en una actividad esponsorizada, tenían una actitud más positiva hacia la marca expuesta que los menos implicados. La actitud hacia el esponsor es definida por Keller (2003) como una evaluación total de la marca y de la organización, y es una medida de la efectividad de la esponsorización (Farrelly et al., 2006; Lacey et al., 2010; Roy, 2011).

Basándose en la teoría de la identidad social, Gwiner y Swanson (2003), extienden el ámbito de investigación de la identificación organizacional, al determinar que aquellos individuos que son fans de un deporte y tienen un alto nivel de identificación con su equipo, presentan, además de un mayor nivel de reconocimiento de patrocinio, una mayor satisfacción y actitudes más positivas que aquellos que no tienen ese alto nivel de identificación. Bajo la misma argumentación, Grohs y Reisinger (2014) consideran que la implicación del fan desarrolla una actitud más positiva hacia la marca del esponsor.

Además, la implicación al ser considerada como una actitud relativamente estable (Havitz y Mannell, 2005) e intrínseca (Huang, 2006), se ha utilizado para describir la relación de importancia que un deporte ha podido alcanzar en la mente de un consumidor (Houston y Rothschild, 1978; Laurent y Kapferer, 1985; Richins y Bloch, 1986; Green y Chalip, 1997). Para Richins et al. (1992) y Alexandris et al (2007), 
altos niveles de implicación de los individuos conlleva una mayor adquisición de información y la participación en el boca a boca.

En esta línea Bennet et al. (2009), sostienen que los consumidores que asisten a un acontecimiento deportivo normalmente están más implicados con ese deporte concreto, y por tanto tienden a asistir más a los eventos relacionados con el mismo. Por ello, los esponsores deben confiar en que aquellos individuos más implicados con el deporte tendrán más posibilidades de asistir a los eventos deportivos. Así esos niveles más altos de asistencia a los eventos, llevan al consumidor a una exposición mayor al evento y esto está positivamente relacionado con la imagen del mismo (Küster et al., 2009). La imagen del evento según Keller (1993), son las percepciones de un evento, reflejado por las asociaciones en la memoria del consumidor. No debemos olvidar, que las percepciones simbólicas y afectivas hacia el evento se transfieren a la marca del esponsor (Küster et al., 2009). De ahí la importancia de la percepción de la imagen del evento por parte del consumidor.

De forma similar, estudios como el de Gilaninia y Abbaszadeh (2011), Turco (1995), Dees et al, (2008) o Yong Jae et al. (2008) muestra como la implicación del fan en el deporte afecta directamente a la conciencia, la imagen y la intención de compra. A mayores niveles de conocimiento y de imagen, se puede predecir un nivel más alto de la intención de compra. Además, la imagen corporativa está directamente influida por el conocimiento del patrocinador (Gilaninia y Abbaszadeh, 2011). Según Yong Jae et al. (2008), la intención de compra puede ser un gran indicador de la eficacia de la esponsorización, sobre todo cuando un esponsor participa en eventos deportivos que muchas veces van dirigidos a los fans de un deporte, llevándoles a compartir una identidad única.

Por último, señalar que multitud de estudios se ha sugerido que la implicación, como constructo, es crucial cuando se consideran la fidelidad hacia a la marca, la compra y/o el consumo de ésta (Bloom, 1981; Bloch y Richins, 1983; Homer y Kahle, 1988; Ashforth y Mael, 1989; Branscombe y Wann, 1991; Schiffman y Kanuk, 1991; Lovelock et al., 1998 Miloch y Lambrecht, 2006; Yong Jae et al., 2008; Filo et al., 2010). 


\subsection{Planteamiento de hipótesis}

De acuerdo con las relaciones expuestas anteriormente entre el nivel de implicación y otras variables que influyen en patrocinio deportivo, se plantean las siguientes hipótesis de estudio.

Basándonos en las conclusiones de Sandler y Shani, (1989) Crimmins y Horn, (1996); Bennet et al. (2009), donde vincula la implicación con el deporte con el visionado de los eventos relacionados con él se plantea como primera hipótesis de esta investigación la siguiente:

H1: Los consumidores más implicados con el deporte verán más eventos relacionados con ese deporte que los que no lo están.

En relación a la implicación del fan con el deporte, según Dees et al. (2008); Yong Jae et al. (2008) y Gilaninia y Abbaszadeh (2011) afecta directamente a la conciencia, la imagen y la intención de compra, por lo que podemos plantear las siguientes hipótesis:

H2a: Los consumidores más implicados con el deporte tendrán una imagen más positiva del esponsor que los que no lo están.

H2b: Los consumidores más implicados con el deporte tendrán más probabilidad de comprar productos relacionados con éste que los que no lo están.

En la revisión de la literatura realizada, se ha mostrado la relación entre la esponsorización y la imagen de un evento; no obstante, no se han encontrado evidencias de estudios que analicen la influencia del nivel de implicación del consumidor con el deporte sobre la percepción de la imagen del evento. Debido a la importancia de este factor, creemos necesario analizar la relación que existe entre ambas variables, por ello planteamos la siguiente hipótesis:

H3: Los consumidores más implicados con el deporte tendrán una imagen más positiva del evento que los que no lo están.

\section{METODOLOGÍA}

\subsection{Elección de la actividad deportiva}


Con la finalidad de analizar la implicación de los individuos sobre la esponsorización deportiva se ha escogido uno de los eventos deportivos más importantes a nivel europeo, la Eurocopa de Fútbol 2012. Como sostiene Fazio (1986), es importante seleccionar un evento que sea muy familiar para los sujetos, porque así el estímulo producirá asociaciones sobresalientes en los recuerdos de los encuestados.

Este evento deportivo tiene un gran seguimiento por parte del consumidor a nivel mundial y en España, concretamente. Los datos de audiencia que se obtuvieron en los partidos en los que no participó la selección española, oscilaron entre el 18,42\% y el $28,98 \%$ de cuota de pantalla (share). Este porcentaje se elevó al $72,7 \%$ cuando jugaba la selección española de fútbol, alcanzando un $83,4 \%$ en la final del campeonato, que enfrentó España con Italia, y tuvo una audiencia de 15.481.000 espectadores ${ }^{3}$.

No hay que olvidar, la importancia económica que supuso la inversión realizada en la Eurocopa de fútbol 2012. El presupuesto de la UEFA para la Eurocopa fue de 1.355 millones de euros, que provenían de los ingresos por patrocinios (290 millones, un $21 \%$ del total), los ingresos por la venta de los derechos televisivos (840 millones, 62\%) y la venta de entradas (225 millones, 16\%).

Y por último, otra importante razón de haber elegido la Eurocopa de fútbol 2012, es que todos equipos contaban con esponsores, y por lo tanto los signos de identificación corporativa del éstos aparecían en pantalla en las imágenes propias de la competición (Lardinoit y Derbaix, 2001).

\subsection{Cuestionario}

Para elaborar el cuestionario se emplearon diferentes escalas previamente desarrollados por otros investigadores. En la mayoría de los ítems se ha utilizado una escala de tipo Likert de 7 puntos, ya que es la más utilizada en Ciencias Sociales (DeSarbo y Harsman, 1985; Johan y Pham y, 1999; Gwinner y Swanson, 2003; Carrillat et al., 2005 y Carrillat et al., 2010).

\footnotetext{
${ }^{3}$ Buenestado, D., 2012. La gran final de la Eurocopa 2012 marca grandes datos en todo el continente. Disponible en: http://www.formulatv.com/noticias/25739/gran-final-eurocopa-marcagrandes-datos-todo-continentel
} 
Para medir el nivel de implicación con el deporte, se empleó la batería de 19 preguntas con diferentes pares de adjetivos empleada por Martensen et al., (2007) para describir el interés por el deporte.

En cuanto a las variables que miden la efectividad de la esponsorización: imagen e intención de compra, se emplearon diferentes escalas. Para medir la imagen del consumidor hacia el esponsor se partió de los trabajos realizados por Sandler y Shani, (1992, 1993), Turco (1995), Nicholls et al, (1999) y Gwinner y Swanson (2003), donde se preguntaron a los sujetos por sus impresiones generales hacia los esponsores a través de un diferencial semántico de siete puntos (Sengupta y Fitzsimons, 2000).

Por último, la imagen del evento y la intención de compra se midió utilizando la batería de preguntas propuesta por Carrillat el al. (2005).

\subsection{Análisis y resultados}

La recogida de datos se realizó a través de un muestreo de conveniencia por medio de un cuestionario auto-administrado, durante el mes de noviembre de 2012. La población seleccionada fueron estudiantes universitarios. El perfil del target seleccionado se ajusta al perfil de máxima audiencia del evento deportivo seleccionado. La audiencia registrada en el partido de la final de la Eurocopa 2012 entre España-Italia fue del $31,7 \%$ para los espectadores situados entre 25 y 44 años de edad, y del 32,1\% para los jóvenes entre 13 y 24 años de edad, siendo éste el porcentaje más alto, y el que se encuentra por tanto dentro del rango de edad de nuestra muestra ${ }^{4}$.

Una vez eliminados los cuestionarios incompletos o errores, se obtuvieron 427 cuestionarios válidos. La muestra obtenida tenía una edad comprendida entre los 18 y los 48 años, de los cuales un $57,2 \%$ eran hombres. La media edad es de 21,8 años, y su interés por el fútbol es de media de 3.98 sobre 7 .

El paquete estadístico utilizado para realizar los análisis es el Spss 15.0.

\footnotetext{
${ }^{4}$ España, tricampeona de leyenda en el partido más visto de la historia: 15.5 millones y $83 \%$ de share. Disponible en: http://www.vertele.com/noticias/espana-tricampeona-de-leyenda-en-el-partidomas-visto-de-la-historia-15-5-millones-y-83-de-share/
} 
Para comprobarla fiabilidad y dimensionalidad de las escalas empleadas se utilizó el coeficiente de alpha de Cronbach y las correlaciones ítem-total. Los coeficientes obtenidos presentan valores extremos entre 0,818 y 0,923 (ver Tabla 1), por lo que están dentro de los niveles aceptables de fiabilidad (Nunnally, 1978; Hair et al, 1999). Por otro lado, las correlaciones ítem-total fueron en todos los casos mayores que 0,3 (mínimo aceptable por Nurosis, 1993). Las cargas factoriales fueron significativas en todos los casos, ya que superan el mínimo aceptable de 0,5 (Hair et al, 1999) (ver Tabla 1). 
Tabla 1: Fiabilidad y dimensionalidad de la escala

\begin{tabular}{|c|c|c|c|c|}
\hline Factor & $\begin{array}{l}N^{\circ} \text { items } \\
\text { finales }\end{array}$ & $\begin{array}{c}\text { Fiabilidad (Alfa de } \\
\text { Cronbach) }\end{array}$ & $\begin{array}{l}\text { Correlación } \\
\text { Ítem-total }\end{array}$ & $\begin{array}{c}\text { Cargas } \\
\text { factoriales }\end{array}$ \\
\hline Implicación con el deporte & 15 & ,924 & $\begin{array}{l}, 836 \\
, 849 \\
, 855 \\
, 847 \\
, 769 \\
, 658 \\
, 785 \\
, 816 \\
, 745 \\
, 791 \\
, 669 \\
, 751 \\
, 755 \\
, 781 \\
, 709 \\
\end{array}$ & $\begin{array}{l}, 826 \\
, 811 \\
, 851 \\
, 887 \\
, 704 \\
, 517 \\
, 713 \\
, 741 \\
, 703 \\
, 701 \\
, 486 \\
, 616 \\
, 723 \\
, 662 \\
, 644 \\
\end{array}$ \\
\hline Imagen de los esponsores & 3 & ,927 & $\begin{array}{l}, 874 \\
, 866 \\
, 810 \\
\end{array}$ & $\begin{array}{l}, 947 \\
, 942 \\
, 913 \\
\end{array}$ \\
\hline Imagen del evento & 3 & ,924 & $\begin{array}{l}, 937 \\
, 881\end{array}$ & $\begin{array}{l}, 836 \\
, 874\end{array}$ \\
\hline $\begin{array}{l}\text { Intención de compra } \\
\text { esponsores }\end{array}$ & 4 & 876 & $\begin{array}{l}, 779 \\
, 779\end{array}$ & $\begin{array}{l}607 \\
607\end{array}$ \\
\hline
\end{tabular}

Fuente: Elaboración propia.

Para contrastar la primera hipótesis planteada, que sostiene que los consumidores más implicados con deporte, en este caso con el futbol, verán más eventos relacionados con dicho deporte, se realizó un análisis t de student. Para ello, la variable implicación fue recodificada en base a los cuartiles de la distribución. Los encuestados cuyo interés se situó en el primer y segundo cuartil se etiquetaron como "baja implicación"; y aquellos cuyo interés por el evento deportivo recaían en los cuartiles tercero y cuarto se etiquetaron con nivel de "alta implicación". Los resultados obtenidos (ver Tabla 2) corroboran el planteamiento propuesto, lo más implicados con el fútbol ven más retrasmisiones deportivas. 
Tabla 2: Pruebas de comparación de medias entre la implicación con el deporte y el visionado de eventos

\begin{tabular}{|c|c|c|c|}
\hline & $\mathrm{M}^{*}$ & $\mathrm{~F}$ & $p$ \\
\hline \multicolumn{1}{|l|}{ Alta implicación deporte } & $5,231(, 839)$ & 3,969 &, 000 \\
\hline \multicolumn{1}{|l|}{ Baja implicación deporte } & $2,667(, 910)$ & $-37,543$ &, 000 \\
\hline $\begin{array}{l}\text { *Entre paréntesis aparece la desviación típica } \\
\text { Fuente: Elaboración propia }\end{array}$ & \\
\hline
\end{tabular}

En la tabla 3, podemos observar los resultados de $\mathrm{T}$ de Student, realizada para contrastar la hipótesis H2a, donde los consumidores más implicados con el deporte tendrán una imagen más positiva del esponsor que los que no lo están. El nivel, de significación $\leq, 000$ entre la diferencias de medias nos indica la existencia de una relación positiva entre la implicación y la imagen del esponsor.

Tabla 3: Pruebas de comparación de medias entre implicación con el deporte y la imagen del esponsor

\begin{tabular}{|c|c|c|c|}
\hline & $M^{*}$ & $\mathrm{~F}$ & $p$ \\
\hline Alta implicación deporte & $5,231(, 839)$ & 3,969 & ,000 \\
\hline Baja implicación deporte & $2,667(, 910)$ & $-37,543$ & ,000 \\
\hline
\end{tabular}

Al igual que en el caso anterior, para contrastar el planteamiento de que aquellos consumidores más implicados con el deporte tendrán una intención de compra mayor que los que no (hipótesis H2b), se realizó un análisis de comparación de medias (prueba $t$ de Student). En ambos casos se obtuvieron valores $p \leq, 000$, por lo que se rechaza la hipótesis nula y podemos asegurar que hay diferencias significativas, y que por tanto aceptamos la hipótesis planteada (ver tabla 4).

Tabla 4: comparación medias entre implicación con el deporte y la intención de compra

\begin{tabular}{|c|c|c|c|}
\hline & $\mathrm{M}^{*}$ & $\mathrm{~F}$ & $p$ \\
\hline Alta implicación deporte & $3,254(1,970)$ & 12,686 &, 000 \\
\hline \multicolumn{1}{|c|}{ Baja implicación deporte } & $2,232(1,065)$ & 13,741 &, 000 \\
\hline $\begin{array}{l}\text { * Entre paréntesis aparece la desviación típica } \\
\text { Fuente: Elaboración propia }\end{array}$
\end{tabular}


Para último, para contratar la hipótesis de que los consumidores más implicados con el deporte tendrán imagen más positiva del evento que los no, se realizó una prueba $\mathrm{t}$ de student. En la tabla 5 podemos observar en como la media de los consumidores con alta implicación con el deporte es muy superior a la media de los consumidores con baja implicación con el deporte, y que existe una relación entre la alta implicación y la imagen más positiva del evento ya que $p \leq, 000$.

Tabla 5: comparación medias entre implicación con el deporte y la imagen del evento

\begin{tabular}{|c|c|c|c|}
\hline & $\mathrm{M}^{*}$ & $\mathrm{~F}$ & $p$ \\
\hline Alta implicación deporte & $5,231(, 839)$ & 3,969 &, 000 \\
\hline Baja implicación deporte & $2,667(, 910)$ & $-37,543$ &, 000 \\
\hline $\begin{array}{l}\text { * Entre paréntesis aparece la desviación típica } \\
\text { Fuente: Elaboración propia }\end{array}$ & \\
\hline
\end{tabular}

\section{4 . Conclusiones, limitaciones y futuras líneas de investigación}

Tras el análisis de los resultados se puede concluir que el nivel de implicación influye en cuatro factores, en el visionado de eventos relacionado con el deporte, la imagen de los esponsores, la imagen del evento y la intención de compra.

En concreto que el visionado del deporte se vea afectado por la implicación con el deporte, es interesante ya que la esponsorización basa su efectividad en el seguimiento del deporte o evento seleccionado. Los espectadores de eventos deportivos están expuestos a los mensajes promocionales bajo unas condiciones favorables, como son el entusiasmo, la excitación y la diversión; condiciones bajo las cuales, los consumidores tienden a relajarse y a ser más receptivos a los mensajes corporativos (Nicholls et al., 1999), esto hace que la esponsorización sea un medio muy eficaz para llegar al consumidor.

En cuanto a la influencia positiva sobre la imagen de los esponsores del nivel de implicación con el deporte, corrobora uno de los objetivos fundamentales de la esponsorización, tal y como sostienen Gilaninia y Abbaszadeh (2011). Por lo tanto, resulta fundamental tener en cuenta la imagen que la empresa esponsorizada quiere dar a la hora de escoger que deporte es el adecuado a esponsorizar. Así mismo, Ferrand y Pages (1999), consideran que la imagen de la empresa, marca o producto 
y la imagen del evento son determinantes en la efectividad de la esponsorización, pues de su interacción derivarán una serie de efectos en la imagen del esponsor; es un elemento que no debemos perder de vista ya que para la construcción de la imagen de la organización es fundamental.

Finalmente, otro de los factores que hemos investigado, y que afecta directamente a los beneficios económicos de la empresa es la intención de compra y su relación con el nivel de implicación. Además, la intención de compra es una medida comportamental, que nos puede ayudar a comprobar la efectividad de la esponsorización (Koo et al., 2005; Yong Jae et al., 2008; Gilaninia y Abbaszadeh, 2011).

Además, los resultados nos dan la posibilidad de segmentar a los consumidores y formular estrategias eficaces de marketing basadas en su nivel de participación. Los consumidores con mayores niveles de participación son más rentables, pueden convertirse en líderes de opinión y pueden obtenerse mejores respuestas a las actividades promocionales como son grandes compradores, apoyado por AlonsoDos-Santos et al. (2016).

En cuanto a las limitaciones del estudio, hay que tener en cuenta que esta investigación ha sido realizada con estudiantes universitarios, con las restricciones que ello conlleva, no obstante muchos de los estudios publicados en este campo han sido realizados sobre el mismo segmento de la población dada la conveniencia de realizar el trabajo de campo sobre la misma (Gwinner y Eaton 1999; Dean 1999, 2002; o Levin et al., 2001).

En último lugar podríamos proponer varias líneas de investigación futura que profundicen en el entendimiento de la efectividad de la esponsorización; por un lado, sería de interés comparar entre diferentes deportes con el fin de comprobar el nivel de implicación de cada uno de ellos, para que éste nos sirviera para dirimir qué deporte seleccionar a la hora de esponsorizar, ya que a mayor implicación más posibilidad de ser vistos. Otra posible línea de investigación sería plantear la hipótesis de la combinación entre el nivel de implicación con el deporte y con el evento, para averiguar cuál de los dos es más determinante a la hora de influir sobre el recuerdo imagen e intención de compra. 


\section{BIBLIOGRAFIA}

Alexandris, K., Tsaousi, E. \& James, J. (2007). Predicting sponsorship outcomes from attitudinal constructs: the case of a professional basketball event. Sport Marketing Quarterly, Vol. 16 No. 3, pp. 130-9.

Alonso-Dos-Santos, M. ; Vveinhardt, J. ; Ferran Calabuig-Moreno, F. \& MontoroRios, J.(2016), Involvement and Image Transfer in Sports Sponsorship, Inzinerine Ekonomika-Engineering Economics, 2016, 27(1), pp.78-89

Ashforth, B., \& Mael, F. (1989). Social identity theory and the organization. Academy of Management Journal, 14, pp.20-39.

Beaton, A. A., Funk, D. C., Ridinger, L., \& Jordan, J. (2011). Sport involvement: A conceptual and empirical analysis. Sport Management Review, 14(2), 126140. http://dx.doi.org/10.1016/j.smr.2010.07.002

Bennett, G.; Ferreira, M.; Lee, J. \& Polite, F. (2009). The role of involvement in sports and sport spectatorship in sponsor's brand use: the case of Mountain Dew and action sports sponsorship. Sport Marketing Quarterly, 2009, 18, pp.14-24.

Bloch, P., \& Richins, M. (1983). A theoretical model for the study of product importance perceptions. Journal of Marketing, 47(3),pp. 69-81.

Bloom, P. (1981). What marketers need to know about the marketing of professional services. In J. H. Donnelly \& W. R. George (Eds.), Marketing of services (pp. 86-87). Chicago: American Marketing Association.

Branscombe, N., \& Wann, D. (1991). The positive social and self-concept consequences of sports team identification. Journal of Sport and Social Issues, 15, pp.115-127.

Buenestado, D., (2012). La gran final de la Eurocopa 2012 marca grandes datos en todo el continente. (recuperado el10-092014): http://www.formulatv.com/noticias/25739/gran-final-eurocopa-marca-grandesdatos-todo-continente/ 
Capella, M. (2002): "Measuring Sports Fans' Involvement: The Fan Behavior Questionnaire", Southern Business Review, Spring, pp.30-36.

Carrillat, F; Lafferty, B. \& Harris, E. (2005). Investigating sponsorship effectiveness: Do less familiar brands have an advantage over more familiar brands in single and multiple sponsorship arrangements?. The Journal of Brand Management, 13 (1), pp.50-64.

Carrillat, F., Harris E. \& Lafferty B. (2010). Fortuitous brand image transfer investigating the side effect of concurrent sponsorships. Journal of Advertising, 39 (2), pp.109-123

Celsi, R.L. \& Olson, J.C. (1988). The Role of Involvement in Attention and Comprehension Processes, Journal of Consumer Research, vol. 15, n², pp.210224.

Chaudhuri, A., (2000). A macro analysis of the relationship of product involvement and information search: the role of risk. Journal of Marketing Theory and Practice, vol. $8, n^{\circ} 1$, pp. 1 - 15.

Crimmins, J. \& Horn, M. (1996). Sponsorship: from management ego trip to marketing success. Journal of Advertising Research, 36 (4), pp.11-21.

D'Astous, A. \& Bitz, P. (1995). Consumer evaluations of sponsorship programmes. European Journal of Marketing, 29 (12), pp.6-22.

Day, E.; Royne, M. \& Camacho, A., (1995). Opportunities for involvement research: a scale development approach. Journal of Advertising, Vol. 24, № 3, otoño, pp. 69-75

Dean, D. H. (1999). Brand endorsement, popularity and event sponsorship as advertising cues affecting consumer pre-purchase attitudes. Journal of Advertising, 28 (3), pp.1-12.

Dean, D. H., 2002. Associating the corporation with charitable event through sponsorship: measuring the effects on corporate community relations. Journal of Advertising. 31(4), pp.77-87.

DeSarbo, W. S. \& Harshman, R. A. (1985). Celebrity-brand congruence analysis. Current Issues and Research in Advertising, 1, pp.17-52. 
Dees,W; Bennett, G. \& Villegas, J. (2008). Measuring the Effectiveness of Sponsorship of an Elite Intercollegiate Football Program. Sport Marketing Quarterly, 2008, 17, pp.79-89,

Diamond, W. D., \& Kashyap, R. K. (1997). Extending models of prosocial behavior to explain university alumni contributions. Journal of Applied Social Psychology, 27(10), pp.915-928.

Donovan, D. T.; Carlson, B. D. \& Zimmerman, M. (2005). The influence of personality traits on sports fan identification. Sport Marketing Quarterly, 14, 1, pp.3142.

Droge, Cornelia (1989). Shaping the route to attitude change: central versus peripheral processing through comparative versus no comparative advertising. Journal of Marketing Research, 26 (May), pp.193-204.

España, tricampeona de leyenda en el partido más visto de la historia: 15.5 millones y $83 \%$ de share. (recuperado el 30-10-2013)

de:: http://www.vertele.com/noticias/espana-tricampeona-de-leyenda-en-el-partidomas-visto-de-la-historia-15-5-millones-y-83-de-share/

Evrard, Y. \& Aurier, P., (1996). Identification and validation of the components of the person-object relationship. Journal of Business Research, vol. 37, pp.127-134.

Farrelly, F. J., Quester, P. G, \& Burton, R. (2006). Changes in sponsorship value: Competencies and capabilities of successful sponsorship relationships. Industrial Marketing Management, 35(8), pp. 10161026. http://dx.doi.org/10.1016/j.indmarman.2006.05.006

Fazio, R. H., 1986. How do attitudes guide behavior? In R. M. Sorrentino E. T. Higgins (Eds.), The handbook of motivation and cognition: Foundations of social behavior. New York: GuilfordPress, pp.204-243.

Ferrand, A. \& Pages, M. (1999). Image management in sport organizations: The creation of value. European Journal of Marketing, 33(3/4), pp.387-401.

Filo, K., Funk, D. C., \& O'Brien, D. (2010). The antecedents and outcomes of attachment and sponsor image within charity sport events. Journal of Sport Management, 24, pp.623-648. 
Fisher, R. \& Wakefield, K. (1998). Factors leading to group identification: a field study of winners and losers". Journal of Psychology \& Marketing, Vol. 15, Enero,pp. 23-40.

Funk, D.; Redinger, L. \& Moorman, A. (2004). Exploring origins of involvement: Understanding the relationship between the consumer motives and involvement with professional sports teams. Leisure Sciences, 26, pp. 35-61.

Galván, I. \& González, J. (2003): El espectáculo deportivo y el espectador, simbiosis de relaciones psicosociales, Lecturas: Educación física y deportes, Nº. 64, 2003

García, P. \& Díaz, A., 2012. Inversión, negocio y algo de fútbol. (recuperado el 1405-2013 ) de: http://www.tiempodehoy.com/espana/inversion-negocio-y-algo-defutbol

García Ferrando, M. \& Llopis, R. (2006). Posmodernidad y deporte: entre la individualización y la masificación. Encuesta de hábitos deportivos de los españoles 2005. CSD. Madrid.

Gilaninia, S. \& Abbaszadeh, M.H., (2011). Assessing the constructs of sport sponsorship effectiveness and theoretical relationships between them among football fans in Iran. Journal of Basic and Applied Scientific Research, 1(7),pp. 606-616

Green, C, \& Chalip, L. (1997). Enduring involvement in youth soccer: The socialization of parent and child. Journal of Leisure Research, pp. 29, 61-

Greenwood, P., Kanters, M. \& Casper, J. (2006). Sport Fan Team Identification Formation in Mid-Level Professional Sport. European Sport Management Quarterly, 6 (3), pp. 253-265.

Greenwald, A. \& Leavitt, C. (1984). Audience Involvement in Advertising: Four Levels. Journal of Consumer Research, 11 (Junio), pp.581-592

Grohs, R., \& Reisinger, H. (2014). Sponsorship effects on brand image: The role of exposure and activity involvement. Journal of Business Research, 67(5), 10181025. http://dx.doi.org/10.1016/j.jbusres.2013.08.008

Gwinner, K. \& Eaton, J. (1999). Building bran image through event sponsorship: the role of image transfer. Journal of Advertising, 28 (4), pp. 47-57. 
Gwinner, K. \& Swanson, S. R. (2003). A model of fan identification: antecedents and sponsorship outcomes. Journal of Services Marketing, 17 (3),pp. 275-294.

Hair, J., Anderson, R., Tatham, R. \& Black, W. (1999). Análisis Multivariante. 5ª ed. Madrid. Editorial Prentice Hall.

Havitz, M. E., \& Dimanche, F. (1997). Leisure involvement revisited: Measurement advances and conceptual conundrums. Journal of Leisure Research , 29, pp. 245278.

Havitz, M. E., \& R. C. Mannell. (2005). Enduring Involvement, Situational Involvement in Leisure and Non-Leisure Activities. Journal of Leisure Research, 37 (2), pp. 152-77.

Homer, P \& Kahle, L. (1989). A Structural Equation Test of the Value-AttitudeBehavior Hierarchy. Journal of Personality and Social Psychology, 54(4), pp.638-646

Houston, M. J. \& Rothschild, M. L. (1978). Conceptual and Methodological Perspectives on Involvement. Educators' Proceedings, ed. S. C. Jain, Chicago: American Marketing Association, pp. 184-187.

Hur, Y., Ko, Y. J., \& Claussen, C. L. (2012). Determinants of using sports web portals: An empirical examination of the sport website acceptance model. International Journal of Sports Marketing \& Sponsorship, 13(3), pp. 169-188.

Johan, G. V. \& Pham, T. M., (1999). Relatedness, prominence, and constructive sponsor identification. Journal of Marketing Research, 3 (3), pp. 299-313.

Kahle, L. R., Kambara, K. M., \& Rose, G. M. (1996). A functional model of fan attendance motivations for college football. Sport Marketing Quarterly, 5(4), pp. 5160.

Keller, K. L., (2003). Conceptualizing, measuring and managing consumer-based brand equity. Journal of Marketing, 57 (1), pp. 1-22.

Kim, Y.; Walsh, P. \& Ross, S. (2008). An Examination of the Psychological and Consumptive Behaviours of Sport Video Gamers. Sport Marketing Quarterly, 2008, 17, Vol. 1, pp. 44-53. 
Ko, Y. J., Kim, Y. K., Kim, M. K., \& Lee, J. H. (2010). The role of involvement and identification on event quality perceptions and satisfaction: A case of US Taekwondo Open. Asia Pacific Journal of Marketing and Logistics, 22(1), pp.25-39.

Koo, G.; Quarterman, J.; Jackson, E. N. \& Suh, Y. (2005). An approach of schematic information processing for sport sponsorship effectiveness. Research Quarterly for Exercise and Sport, 76 (19), pp. 127.

Krugman, H. (1962). An Application of Learning Theory to TV Copy Testing. Public Opinion Quarterly, 26, pp. 626-634.

Küster, I; Vila, N. \& Aldás, J. (2009). Efecto del Patrocinio de laCopa América enlas percepcionesde luis vuitton:una perspectivainternacional. Universia Business Review | Segundo Trimestre

Lacey, R., Close, A. G., \& Finney, R. Z. (2010). The pivotal roles of product knowledge and corporate social responsibility in event sponsorship effectiveness. Journal of Business Research, 63(11), pp.12221228. http://dx.doi.org/10.1016/j.jbusres.2009.11.001

La gran final de la Eurocopa 2012 marca grandes datos en todo el continente. El España-Italia tuvo pendientes a más de la mitad de los espectadores europeos. (recuperado el 15-09-2014) de:: http://www.formulatv.com/noticias/25739/gran-finaleurocopa-marca-grandes-datos-todo-continentel.

Lardinoit, T. \& Derbaix, C. (2001). Sponsorship and recall of sponsors. Psychology \& Marketing, 18 (2), pp. 167-190.

Lascu, D.N., Giese, T.D., Toolan, C., Guehring, B. \& Mercer, J. (1995). Sport involvement: a relevant individual difference factor in spectator sports. Sport Marketing Quarterly, 4 (4), pp. 41-46.

Laurent, G. \& Kapferer, J., (1985). Measuring consumer involvement profiles. Journal of Marketing Research, Vol. 22, Nº 1, febrero, pp. 41-53.

Laverie, D. \& Arnett, D. (2000). Factors affecting fan attendance: The influence of identity salience and satisfaction. Journal of Leisure Research, 32(2), pp. 225-246. 
Levin, A. M.; Joiner, C. \& Cameron, G. (2001). The impact of sports sponsorship on consumer' Brand attitudes and recall: the case of NASCAR fans. Journal of Current Issues and Research in Advertising, 23 (2), pp. 23-31.

Lovelock, C, Patterson, P., \& Walker, R. (1998). Services marketing. Sydney, Australia: Prentice Hall.

Martensen, A., Gronholdt, L., Bendtsen, L., \& Jensen, M. J. (2007). Application of a model for the effectiveness of event marketing. Journal of Advertising Research, 47(3), pp. 283-301. http://dx.doi.org/10.2501/S0021849907070316

Martin, C.L., (1998). Relationship marketing: a high-involvement product attribute approach. Journal of product \& brand management, vol. 7, nº 1,pp. 6-26.

Maheswaran, D. \& Sternthal, B. (1990).The effects of knowledge, motivation and type of message on ad processing and product judgements. Journal of Consumer Research, vol. 17, junio, pp. 66-73.

Mazodier, M. \& Quester, P. (2014). The role of sponsorship fit for changing brand affect: A latent growth modeling approach. International Journal of Research in Marketing, 31(1), pp.16-29.

\section{http://dx.doi.org/10.1016/j.jiresmar.2013.08.004}

Meenaghan, T. (2001). Understanding sponsorship effects. Psychology \& Marketing, 18 (2), pp.95-122.

Miloch, K. S. \& Lambrecht, K.W. (2006). Consumer Awareness of Sponsorship at Grassroots Sport Events. Sport Marketing Quarterly, 15,pp.147-154,

Mittal, B. (1989). A theoretical analysis of two recent measures of involvement. Advances in consumer research, vol. 16, editado por T.K. Srull, Provo (UT), Association for consumer research, pp. 697-702.

Morales Vallejo, P. (2011). Guía para construir cuestionarios y escalas de actitudes. (recuperado el 18-05-20139 de: http://blog.uca.edu.ni/kurbina/files/2011/06/guiaparaconstruirescalasdeactitudes. pdf 
Nicholls, J. A. F., Roslow, S. \& Dublish, S. (1999). Brand recall and brand preference at sponsored golf and tennis tournaments. European Journal of Marketing, 33(3/4), pp. 366-386.

Nunnally, J. (1978). Psychometric Theory. $2^{\mathrm{a} e d .}$ New York. McGraw-Hill.

Nurosis, M. (1993). Spss. Statistical data analysis. Ed. SPSS Inc.

Olson, E.L. \& Thjømøe, H.M. (2003). The effects of peripheral exposure to information on brand preference. European Journal of Marketing, Vol. 37 Iss: 1/2, pp. 243 - 255. http://dx.doi.org/10.1108/03090560310453280

Olson, E., 2010. "Does sponsorship work in the same way in different sponsorship contexts?. European Journal of Marketing, 44 (1/2), pp.180 -199.

Pérez, C. (2006). La implicación del consumidor en la adquisición de bienes y servicios. Revista ESIC Market, №. 123, pp.93-113

Peter, J. Paul \& Jerry C. Olson (1987), Consumer Behavior: Marketing Strategy Perspectives, Homewood, IL: Irwin.

Pham, M.T. (1992). Effects of involvement, arousal, and pleasure on there cognition of sponsorship stimuli. In Sherry, J.F. and Sternthal, B. (Eds), Advances in Consumer Research,Vol.19, Association for Consumer Research , Provo,UT, pp. 85-93.

Quester, P. \& Farrelly, F. (1998). Brand association and memory decay effects of sponsorship: The case of the Australian Formula One Grand Prix. Journal of Product \& Brand Management, $\quad$ 7(6), pp.539-

556. http://dx.doi.org/10.1108/10610429810244693

Quester, P. \& Thompson, B. (2001). Advertising and promotion leverage on arts sponsorship effectiveness. Journal of Advertising Research, 41(1), pp.33-47

Richins, Marsha L., Peter H. Bloch \& Edward F. McQuarrie (1992). How Enduring and Situational Involvement Combine to Create Involvement Responses. Journal of Consumer Psychology, 1, 2, pp. 143-153.

Sherif, M., \& Cantril, H. (1947). The psychology of ego-involvement. New York: John Wiley \& Sons, Inc. 
Rothschild, M.L. (1984). Perspectives on Involvement: Current Problems and Future Directions, Advances in Consumer Research, vol. 11, pp.216-217.

Sandler, D.M. \& Shani, D. (1989). Olympic sponsorship vs ambush marketing-who gets the gold. Journal of Advertising Research, 29 (4) ,pp. 9-14

Sandler, D. \& Shani, D. (1992). The value of sponsorship in sports marketing: an empirical study. In Proceedings of the 1992 Conference of the American Academy of Advertising. August-September, pp. 9-14.

Sandler, D. \& Shani, D. (1993). Sponsorship and Olympic Games: the consumer perspective. Sport Marketing Quarterly, 2 (3), pp.38-43.

Sengupta, J. \& Fitzsimons, G.J. (2000). The effects of analyzing reasons for brand preferences: disruption or reinforcement?. Journal of Marketing Research, 37, pp.318-30.

Shank, M. \& Beasley, F. (1998): "Fan or fanatic: Refining a measure of sports Involvement", Journal of Sport Behaviour, 21(4), pp.435-443.

Schiffman, L.G. \& Kanuk, L. L. (1991). Consumer behavior.. Prentice-Hall International, Business \& Economics - 680 pages.

Stevens, S., \& Rosenberger, P. J. (2012). The influence of involvement, following sport and fan identification on fan loyalty: An Australian perspective. International Journal of Sports Marketing \& Sponsorship, 13(3), pp. 220-234.

Stone, R. N. (1984). The Marketing Characteristics of Involvement. in NA - Advances in Consumer Research Volume 11, eds. Thomas C. Kinnear, Provo, UT : Association for Consumer Research, pp. 210-215.

Turco, D., 1995. The influence of sponsorship on product recall and image among sport spectator. In world marketing congress proceedings. Academy of Marketing Science, 7 (3), pp. 116-110.

UEFA thanks partner Ford. (recuperado el 23-04-2015) de: http://www.uefa.org/about$\underline{\text { uefa/administration/marketing/news/newsid=2069538.html }}$ 
EL NIVEL DE IMPLICACIÓN DEL CONSUMIDOR... Soledad Zapata Agüera y Laura Martínez Caro

Van Heerden, C. H., (2001). Factors affecting decision-making in South African sport sponsorship. Tesis, (Dr). University of Pretoria. South Africa. (recuperado el 23-042015)

de: http://repository.up.ac.za/bitstream/handle/2263/29282/Complete.pdf?sequence= $\underline{15}$

Wann, D. \& Schrader, M. (2000). Controllability and stability in the self-serving attributions of sport spectators. The Journal of Social Psychology 140(2), pp.160-168 Wolfsteiner, E., Grohs, R. \& Wagner, U. (2015). What drives ambush marketer misidentication?. Journal of Sport Management, 15(4), pp.367-383

Wright, P. L. (1973). The cognitive processes mediating acceptance of advertising. Journal of Marketing Research, 53-62., 1 (4), pp.38-47.

Yong Jae, K., Kyoungtae, K., Cathryn L,C. \& Tae Hee, K. (2008).The effects of sport involvement, sponsor awareness and corporate image on intention to purchase sponsors' products. International Journal of Sports Marketing \& Sponsorship, January 01.

Zaichkowsky, J. (1985).Measuring the involvement construct. Journal of Consumer Research, 12, pp.341-352. 